\title{
The Dimensionless-Parameter Robust Optimization Method Based on Geometric Approach of Pulley Block Compensation In luffing Mechanism
}

\author{
Y. Xue, M.S. Ji, N. Wu \\ School of Mechatronics Engineering \\ Harbin Institute of Technology \\ Harbin, China
}

\author{
Y. Xue, W. Wang \\ School of Civil Engineering \\ Harbin Institute of Technology \\ Harbin, China
}

\begin{abstract}
When using the minimum unbalance torque as the objective function to establish the optimization mathematical model of pulley block compensation in luffing mechanism, the dimensional optimal solutions do not have extension applicability. To solve this problem, a new optimization mathematical model, using the horizontal trajectory deviationas function, was established through geometric method. The working length of the movable arm was taken as measurement, and the geometric parameters of the mechanism were transformed into dimension less parameters, and the function was linearized. As the mathematical model was solved, this article got a universal dimensionless optimal solution which is suit to different kinds of luffing mechanism. The application example shows that the optimal result using this method is obviously better than that using the minimum unbalanced moment as the objective function.
\end{abstract}

Keywords-geometric method; luffing jib; pulley block compensation; dimensionless-parameter; optimization design

\section{INTRODUCTION}

Jib luffing is a kind of luffing method, which is widely used in the luffing jib tower crane and portal crane[1].During the luffing process, the gravity center of heavy will be lifting. The change of the height of it will cause energy waste, greater inertia load and structure vibration, which will lower the crane efficiency. Compensation assembly pulley mechanism is an effective method to reduce the change of the gravity center of heavy, caused by jib luffing. The principle is to use the height change of pulley block in luffing process to compensate the ascending (or descending) height of heavy center. The core problem is to find suitable pulley block pivot position, under the given structure geometric parameters and rate conditions. The graphic method is a convenient method to approximate the position, but its design precision is poor. The optimization method is a relatively accurate method for solving, but establishing the optimization model and solving the optimal function make design work more complicated. Such as Literature [2], [3], they established the objective function with unbalanced torque of the heavy, and used robust optimization to get results. These optimal solutions do not have extensive applicability, and they can only apply to a specific luffing mechanism which some design variablesare given. To solve this problem, this paper used geometric programming approach, and established a new optimization function, using the horizontal trajectory deviation as function. The geometric parameters of the mechanism were dimensionless processing and linear processing. Through such optimization design, the results can be applied to different sizes and different specifications of luffing jib cranes.

\section{MATHEMATICAL MODEL}

The key problem of pulley compensation is to determine the location of the compensation fixed pulleys[4]. Reference to the different types of luffing jib cranes using pulley compensation, the geometric model can be simplified as shown in Figure 1. In Figure 1, $\mathrm{O}$ point is the origin of the coordinate system, point $\mathrm{A}$ is the position of fixed pulley, point $\mathrm{B}$ is the position of movable pulley, and point $\mathrm{C}$ is the location of hanging point. The concrete structures of different types of pulley compensated luffing jib cranes are not the same[5]. So $\mathrm{OC}$ line, from the rotation point $\mathrm{O}$ to hanging point $\mathrm{C}$, is taken as honorary rod length to make the geometric model have broader applicability.

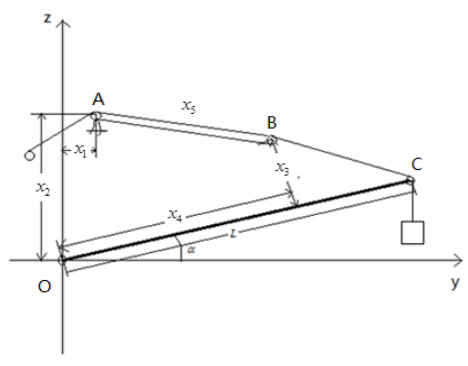

FIGURE I. GEOMETRIC SKETCH OF LUFFING JIB

A. Design Parameters

Known parameters: $L=1$

Design variables: $x_{1}, x_{2}, x_{3}, x_{4}, x_{5}$ (such as Fig. 1, $x_{1}, x_{2}$ determine the coordinates of point $\mathrm{A},{ }^{X_{3}},{ }^{X_{4}}$ determine the coordinates of point $\mathrm{B},{ }^{X_{5}}$ is the rate of compensation pulley block rates and lifting pulley block rates) 
Other parameters: $\alpha$ is the angle between the honorary rod and $\mathrm{x}$-axis, $\alpha_{1}$ is the angle between the honorary rod and $\mathrm{x}$-axis when point $\mathrm{C}$ roses to the highest point

\section{B. Objective Function}

The minimum horizontal displacement deviation of heavy during the whole process is taken as objective function.

Horizontal displacement deviation:

$$
\Delta h=H-\Delta K
$$

$\Delta h$ _ the height change of the heavy center,

$H$ _ the height change of point $\mathrm{C}$

$\Delta K$ — the length change of wire rope $\mathrm{AB}$

Objective function: $\min f=\int_{\alpha_{\min }}^{\alpha_{\max }}|\Delta h| \mathrm{d} \alpha$

Coordinates:

$A:\left(x_{1}, x_{2}\right)$

$B:\left(x_{4} \cos \alpha-x_{3} \sin \alpha, x_{4} \sin \alpha+x_{3} \cos \alpha\right)$

$C:(L \cos \alpha, L \sin \alpha)$

Removing dimension ( $L=1$ );

Coordinates:

$A:\left(x_{1}, x_{2}\right)$

$B:\left(x_{4} \cos \alpha-x_{3} \sin \alpha, x_{4} \sin \alpha+x_{3} \cos \alpha\right)$

$C:(\cos \alpha, \sin \alpha)$

The variable quantity of $\mathrm{C}$ point:

$$
H=\sin \alpha-\sin \alpha_{1}
$$

$$
\begin{gathered}
K=\sqrt{\left(x_{4} \cos \alpha-x_{3} \sin \alpha-x_{1}\right)^{2}+\left(x_{4} \sin \alpha+x_{3} \cos \alpha-x_{2}\right)^{2}} \\
\alpha=\alpha_{1}: \\
K_{1}=\sqrt{\left(x_{4} \cos \alpha_{1}-x_{3} \sin \alpha_{1}-x_{1}\right)^{2}+\left(x_{4} \sin \alpha_{1}+x_{3} \cos \alpha_{1}-x_{2}\right)^{2}}
\end{gathered}
$$

Rope length remains unchanged:

$$
\Delta K=\left(K_{1}-K\right) x_{5}
$$

$$
\begin{gathered}
\Delta h=\left(\sin \alpha-\sin \alpha_{1}\right)-x_{5}\left(\sqrt{\left(x_{4} \cos \alpha_{1}-x_{3} \sin \alpha_{1}-x_{1}\right)^{2}+\left(x_{4} \sin \alpha_{1}+x_{3} \cos \alpha_{1}-x_{2}\right)^{2}}\right) \\
\left.-\sqrt{\left(x_{4} \cos \alpha-x_{3} \sin \alpha-x_{1}\right)^{2}+\left(x_{4} \sin \alpha+x_{3} \cos \alpha-x_{2}\right)^{2}}\right)
\end{gathered}
$$

In operation, the lifting point $\mathrm{C}$ is in the allowable range of deviation

$$
h=\max \Delta h-\min \Delta h \leq \delta
$$

$\delta$ the maximum allowable height deviation $\delta=0.05\left(R_{\max }-R_{\text {min }}\right)$
$R_{\max }, R_{\min } \quad$ maximum, minimum luffing amplitude

In the actual calculation, the luffing amplitude is substituted with $\mathrm{n}$ discrete points, andthe summation of all the points should be the minimized.

Objective function:

$$
\min f=\frac{\Sigma \sqrt{\Delta h^{2}}}{n}
$$

Due to the highly nonlinear function between design and design variables, it is hard to optimize such function[6]. As the design variable deviation $\Delta x$ is small, and function value changes continuously with $x$, nonlinear function $f(x, y)$ can be converted to a linear function. Nonlinear function of the design variables $L_{x}$ and $L_{y}$ in the tiny field is expanded into a Taylor series, and takes the third-order item as a first approximation, while the higher-order terms are ignored[7]. Then, Eq.9 can be replaced by linear formula Eq.10

$$
f=f\left(x_{1}, x_{2}, x_{3}, x_{4}, x_{5}\right)+\sum_{i=1}^{n}\left(\frac{d f}{d x_{i}}\right) d x_{i}
$$

\section{Constraints}

Constraint function:

$$
g=h-\delta<0
$$

In the design of luffing mechanism, the main constraint is built mainly based on experience and the overall arrangement to determine the range of the design variables. In order to ensure optimal usability, the boundary constraints of the design variables:

$$
\begin{gathered}
-0.2 \leq x_{1} \leq 0.2, \quad 0.1 \leq x_{2} \leq 0.6, \quad 0 \leq x_{3} \leq 0.15, \\
0.4 \leq x_{4} \leq 1, \quad 1 \leq x_{5} \leq 4
\end{gathered}
$$

\section{OPTIMUM SOLUTION}

In the actual calculation, the luffing amplitude range is replaced by 21 discrete points. Genetic algorithms are used in optimization process[8]. Genetic algorithms are simulated biological evolution to search for an optimal solution by the method. Optimization results are shown in Table 1

TABLE I. OPTIMIZATION RESULTS

\begin{tabular}{|l|l|l|l|l|l|}
\hline Parameters & $x_{1}$ & $x_{2}$ & $x_{3}$ & $x_{4}$ & $x_{5}$ (ratio) \\
\hline Results & -0.0073 & 0.3092 & 0.1624 & 0.986 & $2.9962 \approx 3$ \\
\hline
\end{tabular}

This method is dimensionless geometric optimization, the resulting geometry optimization results $X_{1}, x_{2}, x_{3}, x_{4}$ are the ratio of the arm length $\mathrm{L}$ and reputation. Thus designers can directly refer to this 
optimization results to optimize the Pulley compensation mechanism.

Vertical trajectory of the center of gravity of the cargo during luffing is shown as Fig.2

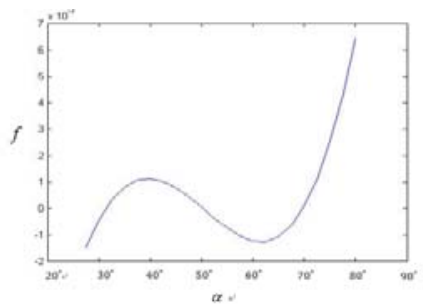

FIGURE II. VERTICAL TRAJECTORY OF THE CENTER OF GRAVITY OF THE HEAVY

Abscissa $\alpha$ is amplitude angle, Ordinate $f_{\text {is }}$ the displacement of the center of gravity of the weight (the ratio of actual displacement and honorary rod length). The figure shows that the optimization results have high accuracy. Heavy focus keeps horizontal during luffing process[9].

\section{EXAMPLE ANALYSIS}

The main technical parameters of a crane: jib length $L=8000 \mathrm{~mm}$, lifting capacity $Q=1 t$, hoisting torque $M=40 \mathrm{KN} \cdot \mathrm{m}$

The selection of the design variables:

$X=\left\{x_{1}, x_{2}, x_{3}, x_{4}, x_{5}\right\}$, these variables are shown in Figure.1

\section{(2) Optimization results}

The results of optimal values are obtained from the former Dimensionless optimization results as shown in Table. 1

$$
x_{1}=-0.0073 \mathrm{~L}, x_{2}=0.3092 L, x_{3}=0.1624 L, x_{4}=0.986 L, x_{5}=3
$$

The actual optimization results was obtained, when the actual value of $\mathrm{L}$ was put into the eq.12. The result is shown in Table.2.

TABLE II. DIMENSIONLESS OPTIMIZATION RESULTS

\begin{tabular}{|c|c|c|c|c|c|}
\hline & $\begin{array}{c}x_{1} / \\
\mathrm{mm}\end{array}$ & $\begin{array}{c}x_{2} / \mathrm{m} \\
\mathrm{m}\end{array}$ & $\begin{array}{c}x_{3} / \mathrm{m} \\
\mathrm{m}\end{array}$ & $\begin{array}{c}x_{4} / \mathrm{m} \\
\mathrm{m}\end{array}$ & $\begin{array}{c}x_{5} \text { (Magnificatio } \\
\mathrm{n} \text { ratio) }\end{array}$ \\
\hline $\begin{array}{c}\text { dimensionless } \\
\text { optimization }\end{array}$ & $\begin{array}{c}-58 . \\
4\end{array}$ & 2473.6 & 1299.2 & 7888 & 3 \\
\hline
\end{tabular}

In order to verify the method of reliability, given the known magnification ratio $\times 5=3$ as the premise, this paper compared this method withthe robust optimizationmethod in the literature [2]. The results of two kinds of optimization in the literature [2] are shown in Table.3. Figure 3 shows the geometric parameters, obtained by this method, make the height variation more close to horizontal line.

TABLE III. MINIMUM TORQUE OPTIMIZATION RESULTS

\begin{tabular}{|l|l|l|ll|l|l|}
\hline & $\begin{array}{c}x_{1} \\
\mathrm{~mm}\end{array}$ & $\begin{array}{c}x_{2} / \mathrm{m} \\
\mathrm{m}\end{array}$ & $\begin{array}{c}x_{3} / \mathrm{m} \\
\mathrm{m}\end{array}$ & $\begin{array}{c}x_{4} / \mathrm{m} \\
\mathrm{m}\end{array}$ & $\begin{array}{c}x_{5} \text { (Magnificatio } \\
\mathrm{n} \text { ratio) }\end{array}$ \\
\hline $\begin{array}{l}\text { robust } \\
\text { optimization }\end{array}$ & -124 & 2031 & 982 & 7240 & 3 \\
\hline
\end{tabular}

The figure of Horizontal trajectory deviation in luffing process

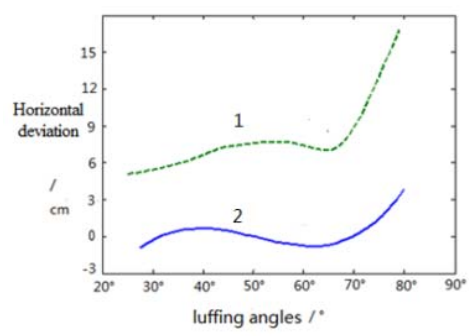

FIGURE III. OPTIMIZATION RESULTS COMPARISON

1-the optimization result of using minimum torque as the objective function

2-the optimization result of geometric method

\section{V.CONCLUSION}

This paper established the optimization mathematical model, using the horizontal trajectory deviation as function, through geometric method. All the geometric parameters are dimensionless parameters. The optimal results were obtained through mathematical method of genetic algorithms[10]. Via example analysis, this article compared this optimization method with other methods which use minimum torque as function and draw some conclusions as following:

Simple mathematical model, less design variables;

The parameters of the mathematical model are dimensionless, which show the position relationships of compensating pulley in luffing jib mechanism. These parameters is irrelevant with the specific size. So they have universality and strong practicability.

The optimization of parameters have high accuracy.The example shows that the optimization parameters gained by this method are better than the unbalance torque method.

The optimization method and the optimized parameters can provide the theoretical basis for the design of pulley block compensation in luffing mechanism.

\section{ACKNOWLEDGEMENTS}

DIY project of Natural Science Foundation of Heilongjiang Province(E201433) 
The fundamental research funds for the Central Universities(HIT.KLOF.2010054)

\section{REFERENCES}

[1] XIE Neng-gang, Wang Qi-ping. Fuzzy Optimization Genetic Algorithm Compensation Pulley Luffing Mechanism Based on Multi-objective[J]. Mechanical transmission.2003.01

[2] CHEN Jin-fu, XUE Long. Physical Planning Act and Its Application in Luffing Mechanism Robust Optimization [J]. Coal Mine Machinery, 2008,29(7):26-29

[3] CHEN Jin-fu, LI Jun. Optimal Design Robustness Compensation Pulley Luffing Mechanism-based Design [J]. Lifting and transport machinery, 2009,03(1): 34-37

[4] Kim,Dong Soo,Lee,Jongsoo. StructualDesign of a Level-luffing Crane Through Trajectory Optimization and Strength-based Sized Optimization[J]. Structural and Multidisciplinary Optimization, July 31,2014: 112-116

[5] J.A.Wright, H.A.Loosemore,R.Farmani. Optimization of Building Thermal Design and ControlBy Multi-criterion Genetic Algorithm Energy and Bulidings, 34(2002), pp.959-972

[6] WU Zhi-Qiang. Reliability Compensation Pulley Luffing Mechanism Robust Optimization Design [J], Coal Mine Machinery,2011,15:21-25

[7] MIAO Ming, SUN Hong-Wei. Optimal Design Method of Compensating Pulley Luffing System [J], Dalian University of Technology

[8] Huang Ying, Yu Wen-hua. Research on robust design optimization method based on MATLAB [J], Journal of Beijing University of Technology, July 23, 2012

[9] Yun Han; Raghavendra Murthy; Marc P. Mignolet; Jeff Lentz. Optimization of Intentional Mistuning Patterns for the Mitigation of the Effects of Random Mistuning [J], Eng. Gas Turbines Power. 2014; 136(6)

[10] C. Bakker; G. T. Parks; J. P. Jarrett.Optimization Algorithms and ODE's in MDO. Design Automation Conference, Volume 3B: 39th 